\title{
Evaluación de Riesgos de Manipulación Repetitiva a Alta Frecuencia Basada en Análisis de Esfuerzos Dinámicos en las Articulaciones sobre Modelos Humanos Digitales
}

\section{RISK ASSESSMENT OF HIGH FREQUENCY REPETITIVE HANDLING BASED ON DYNAMIC STRESS ANALYSIS IN JOINTS OF DIGITAL HUMAN MODELS}

José Javier Marín Zurdo', María Jesús Boné Pina ${ }^{2}$, Carlos Benito Gil ${ }^{3}$

1. Prof. Universidad de Zaragoza (UZ). Coord. Grupo IDERGO.I3A.UZ. Escuela de Ingeniería y Arquitectura. e-mail: jjmarin@unizar.es

2. Médico Residente de Medicina del Trabajo (FREMAP). Técnico Sup. PRL. Grupo IDERGO.I3A.UZ. e-mail: mjbone@unizar.es

3. Ingeniero. Técnico Sup. PRL. Grupo IDERG0.I3A.UZ. e-mail: idergo04@unizar.es

\begin{abstract}
RESUMEN
El nuevo método desarrollado está dirigido a evaluar el riesgo derivado de la realización de tareas repetitivas a alta frecuencia. Utiliza un sistema de captura de movimiento basado en sensores inerciales, utilizable en los propios puestos de trabajo, que nos permite trasladar el movimiento a un modelo biomecánico de antropometría similar al sujeto observado. Incorpora un motor de cálculo de esfuerzos en las articulaciones, que tiene en cuenta las dimensiones antropométricas, las fuerzas externas, las reacciones en los puntos de apoyo, así como las fuerzas de inercia derivadas de las aceleraciones lineales y angulares alcanzadas durante la tarea.

El resultado es un método predictivo de riesgo musculoesquelético que, a diferencia de otros métodos que requieren un trabajo muy minucioso (UNE 1005-3) a la hora de definir las acciones técnicas, reduce drásticamente el proceso de análisis ya que el proceso es automático, y no está influenciado por la subjetividad del evaluador.
\end{abstract}

(Marín J, Boné M, Gil C, 2013. Evaluación de Riesgos de Manipulación Repetitiva a Alta Frecuencia Basada en Análisis de Esfuerzos Dinámicos en las Articulaciones sobre Modelos Humanos Digitales. Cienc Trab. May-Ago; 15 [47]: 86-93).

Palabras clave: EVALUACIÓN RIESGOS LABORALES. MOVIMIENTOS REPETITIVOS. CAPTURA DE MOVIMIENTO HUMANO. SIMULACIÓN CON MODELOS HUMANOS. ESFUERZOS DINÁMICOS EN ARTICULACIONES.

\section{ABSTRACT}

The new method developed is aimed at assessing the risk from repetitive tasks at high frequency. It uses a motion capture system based on inertial sensors for use in their own jobs, which allows us to transfer the movement to a biomechanical model similar to the observed subject anthropometry. Incorporates a calculation engine of efforts in joints, considerating the anthropometric dimensions, external forces, the reactions in the support points, as well as the inertial forces arising from linear and angular accelerations encountered during the task.

The result is a musculoskeletal risk predictive method which, unlike other methods that require a very thorough job (UNE 1005-3) for defining the technical actions, dramatically reduces the analysis process because the process is automated, and is not influenced by the subjectivity of the evaluator.

Key words: OCCUPATIONAL RISK ASSESSMENT, REPETITIVE MOVEMENTS, HUMAN MOTION CAPTURE, SIMULATION WITH HUMAN MODELS. DYNAMIC STRESSES IN JOINTS.

\section{INTRODUCCIÓN}

El nuevo método denominado MoveHuman-Forces ha sido desarrollado por el equipo de Investigación del Prof. Marín de la Universidad de Zaragoza, y está dirigido a evaluar el riesgo musculoesquelético derivado de la realización de tareas repeti-

Correspondencia / Correspondence:

José Javier Marín Zurdo

Maria de Luna, 3, 50018

Zaragoza, España

Tel.: +34 976761978

e-mail: jjmarin@unizar.es

Recibido: 17 de Junio 2013 / Aceptado: 24 de Julio 2013 tivas a alta frecuencia, característico de entornos de producción con ciclos de fabricación cortos. Aunque ya existen métodos enfocados a este propósito (UNE 1005-3)1', su aplicación no está exenta de una cierta subjetividad por parte del evaluador a la hora de contar manualmente -visualizando repetidas veces la filmación realizada en planta- las acciones técnicas llevadas a cabo por el trabajador en cada ciclo de fabricación y los factores de riesgo asociados (sin contar el tiempo que lógicamente requiere este trabajo tan minucioso).

El riesgo de lesión en una articulación depende en gran medida del número de cambios de giro en esa articulación durante la realización de las tareas repetitivas y de factores de riesgo asociados a cada cambio de giro, relativos a magnitud del ángulo, velocidades angulares alcanzadas, fuerzas y/o momentos soportados en la articulación en ese instante, entre otros. Consecuentemente, si fuéramos capaces de detectar esos instantes, donde cada articula- 
ción realiza un cambio de giro y cuantificar los factores de riesgo asociados, podríamos predecir posibles lesiones musculoesqueléticas. Esa es precisamente la base del método MH-Forces.

Para la aplicación del método se hace uso de un sistema de captura de movimiento utilizable en los propios puestos de trabajo, basado en sensores inerciales que se fijan sobre la ropa del trabajador, el cual nos permite trasladar el movimiento capturado a un modelo biomecánico de antropometría similar al sujeto observado. Asimismo, el sistema incorpora un motor de cálculo de esfuerzos en las articulaciones, que tiene en cuenta las dimensiones antropométricas del modelo, fuerzas externas realizadas por el trabajador en cada instante, fuerzas de reacción en los puntos de apoyo (en distintas situaciones, sentado o de pie con uno o dos pies apoyados, o con alguna mano apoyada), y considera también las fuerzas de inercia sobre los centros de gravedad de los distintos segmentos corporales, derivadas de las aceleraciones lineales y angulares alcanzadas durante la tarea.

Fruto de ese motor de cálculo es posible obtener un riesgo asociado a cada articulación y en cada instante donde se produce un cambio de giro: la suma de esos riesgos será el riesgo total asociado a las distintas articulaciones. En consecuencia, el método MH-Forces es un método predictivo de riesgo musculoesquelético de la extremidad superior que agiliza significativamente el proceso de análisis, al no requerir tareas manuales y, a la vez, evita la subjetividad del evaluador.

\section{ANTECEDENTES}

En la actualidad existen distintos métodos de evaluación ergonómica. ${ }^{2} \mathrm{Al}$ respecto podemos citar la ecuación de $\mathrm{NIOSH}^{3,4}$ para la evaluación de tareas de manejo manual de cargas, o el método $\mathrm{REBA}^{5-6}$, que analiza los riesgos musculoesqueléticos derivados de la postura y carga (Fig. 1).

Para el análisis ergonómico de tareas repetitivas podemos destacar el método recogido en la norma UNE-1005-03. ${ }^{1}$ Esta norma europea presenta una serie de pautas en la evaluación y el control de los riesgos para la salud y seguridad debidos a la manipulación repetitiva a alta frecuencia de los miembros superiores en relación con las máquinas. En este punto también podemos destacar un método similar denominado OCRA. ${ }^{7,8}$ La citada norma se basa en determinar un índice de riesgo que se obtiene dividiendo el número de acciones técnicas por el número de acciones técnicas recomendadas.

Las acciones técnicas hay que determinarlas en función de las acciones que realiza el trabajador en cada ciclo de fabricación. La norma incluye recomendaciones a la hora de determinar dichas acciones técnicas. No obstante, se requiere visualizar repetidas veces la filmación del puesto hasta definir con precisión el número de acciones que realiza con cada una de las manos. Se comprende que esta tarea puede requerir un trabajo considerable por parte del técnico de prevención, que dependerá del número y complejidad de las operaciones implicadas en el ciclo de trabajo repetitivo.

Teniendo en cuenta el conjunto de factores que considera este método, sin duda es un método adecuado para evaluar el tipo de actividad que nos ocupa, caracterizada por tareas que se repiten sistemáticamente en cada ciclo de fabricación y donde hay que procurar que el trabajador no supere un número de acciones técnicas en dichos ciclos, que le pueda provocar trastornos musculoesqueléticos a mediano y largo plazo.
Figura 1.

Métodos de Evaluación Ergonómica. Ecuación NIOSH. Carga Postural REBA.

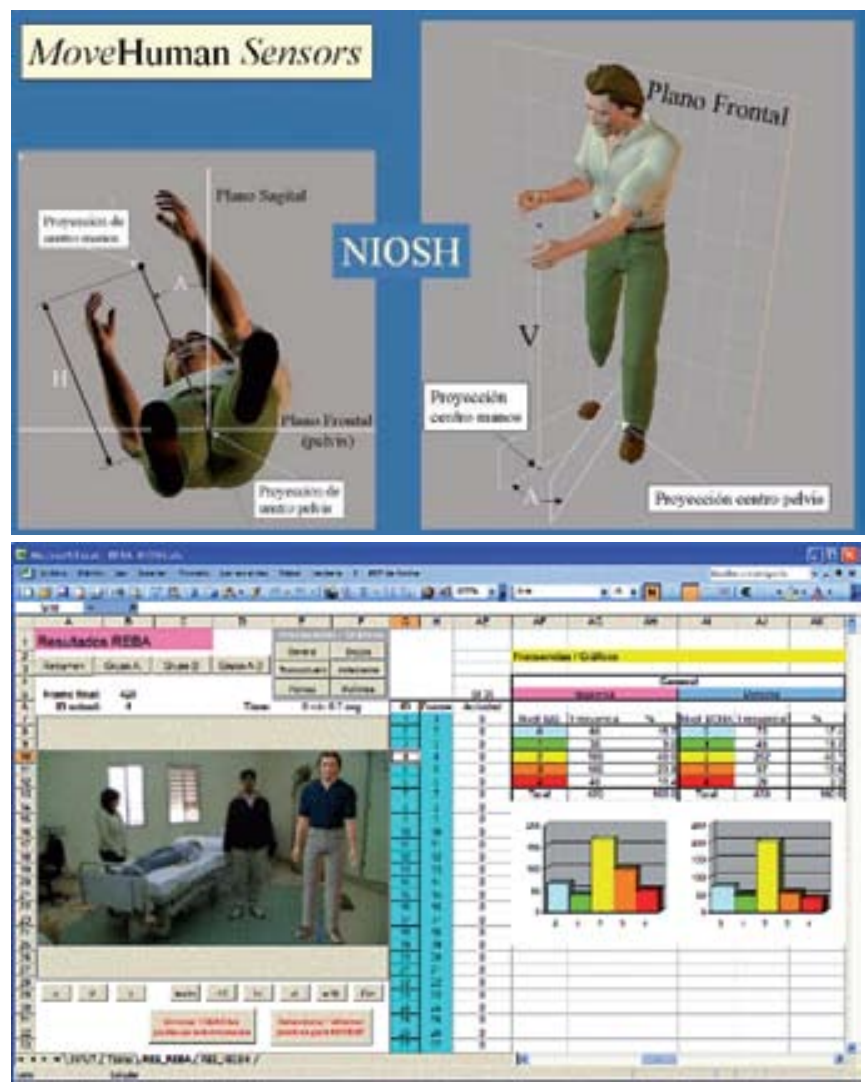

No obstante la aplicación de este método, aún utilizando un software especifico, resulta bastante tedioso, especialmente a la hora de definir cada una de las acciones técnicas, lo cual puede conllevar varias horas de trabajo por parte del técnico de prevención, sin contar el tiempo para redactar el informe de conclusiones o estudiar las posibles acciones correctoras en caso de que fueran necesarias.

Otra cuestión a destacar de la aplicación de este método es la relativa al grado de subjetividad que supone la determinación de ciertas acciones técnicas, algunas de las cuales, atendiendo a los criterios recogidos en la norma, pueden conducir a interpretaciones diferentes. Esto puede dar lugar a que un mismo puesto de trabajo evaluado por dos técnicos de prevención puedan conducir a resultados distintos, ya que la base del método se fundamenta en determinar el número de acciones técnicas realizas en cada ciclo de fabricación, las cuales pueden variar según la interpretación de uno u otro técnico.

Con el fin de superar las dificultades de aplicar un método como el indicado, y aprovechando las posibilidades que ofrecen los sistemas de captura de movimiento actuales ${ }^{9}$ y modelos humanos avanzados $^{10}$ sobre los cuales se pueden aplicar métodos de cálculo biomecánicos en entornos 3D11-13, se ha desarrollado un nuevo método de análisis de riesgos de tareas repetitivas que, en manos del técnico de prevención, va a posibilitar una valoración de este tipo de puestos más ágil y eficiente que los métodos tradicionales, a la vez de reducir drásticamente la subjetividad del evaluador. 


\section{SISTEMA DE CAPTURA DE MOVIMIENTO}

Uno de los elementos claves de cara a la aplicación del método que nos ocupa es la utilización de un sistema de captura de movimiento que nos permita trasladar el movimiento del trabajador a un modelo humano de una antropometría similar al sujeto observado.

Dicha recreación del movimiento nos brinda la posibilidad de aplicar un cálculo biomecánico en cada postura capturada y, en consecuencia, realizar un análisis cinemático de velocidades y aceleraciones lineales y angulares de los segmentos corporales, así como de esfuerzos dinámicos resultantes en las articulaciones derivados del movimiento que realiza el trabajador y de las fuerzas que ejerce sobre los objetos que manipula, información necesaria para la aplicación del método que nos ocupa.

Según lo expuesto, se desprende la necesidad de contar con un sistema de captura de movimiento y, si es posible, aplicable en los propios puestos de trabajo. Para tal propósito se dispone del sistema Move-Human Sensors ${ }^{14}$ (Fig. 2a), desarrollado desde la Universidad de Zaragoza, el cual permite capturar el movimiento humano fuera del entorno de laboratorio, y está dirigido al análisis musculoesquelético de la actividad laboral en condiciones reales de trabajo.

Figura 2.

Fig 2. Esquema funcional de MH-Sensors (a). Componentes del Sistema (b).
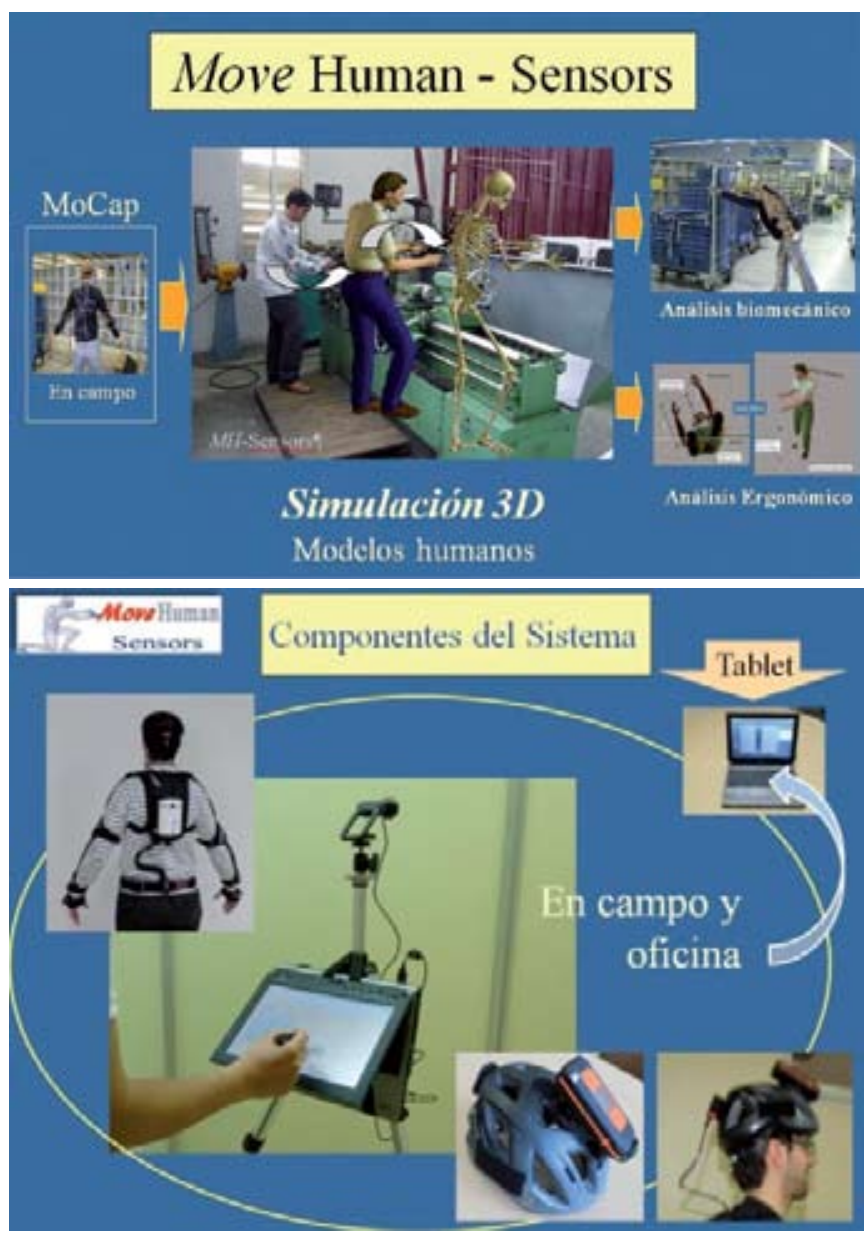

Figura 3.

Sensores Inerciales de Movimiento. Opción Normal y Wireless.
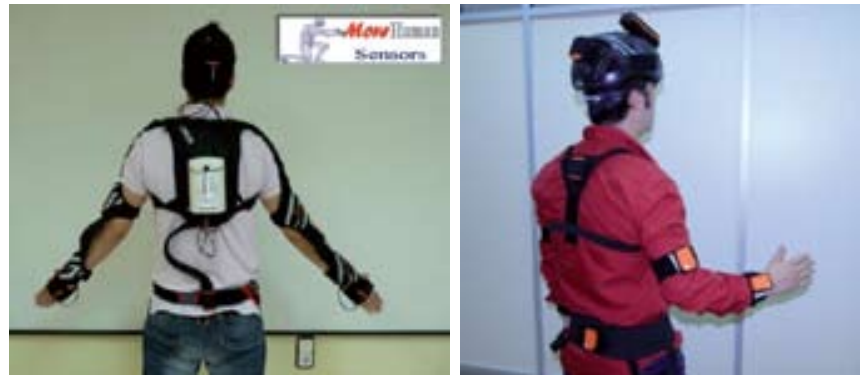

Figura 4.

Visualización de cámara panorámica en caso. Puesto de trabajo simulado.

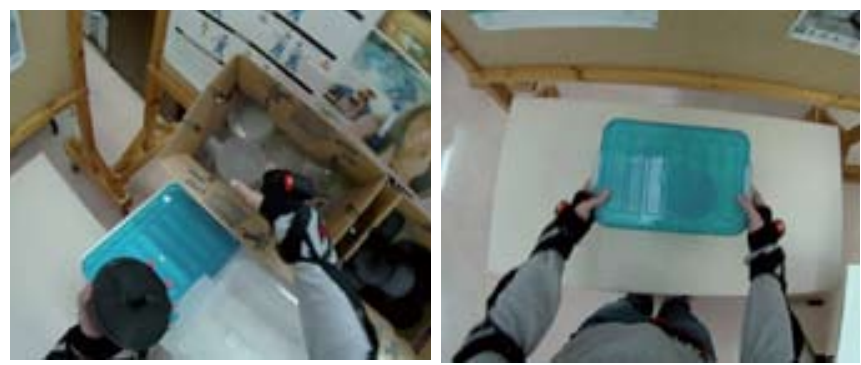

Tecnológicamente está basado en sensores de movimiento inerciales, los cuales se fijan sobre la ropa del trabajador por medio de sujeciones especiales en determinadas puntos anatómicos y están conectados a un concentrador que se ubica en una pequeña mochila que lleva el trabajador en su espalda (Fig. 3).

El sistema se complementa con un ordenador portátil y una cámara tipo webcam, que son los elementos que se precisan para realizar la captura en campo (Fig. 2b).

El trabajador también puede llevar un casco ligero, el cual dispone de una fijación para ubicar el sensor de la cabeza y una sujeción especial para ubicar una cámara panorámica de $170^{\circ}$ que se controla remotamente. La filmación tomada por esta cámara recogerá fielmente las tareas que realiza el trabajador durante la captura, especialmente los movimientos que realice con las manos (Fig. 4), que será importante de cara a la aplicación de un método como 0CRA7, ya citado previamente.

En ciertos casos singulares es posible realizar un estudio donde se combine el sistema de captura de movimiento MH-Sensors con un sistema EMG de superficie wireless (Fig. 5), utilizable también en entornos reales y fuera de condiciones de laboratorio.

Gracias a su portabilidad y facilidad de uso permite realizar evaluaciones en lugares sin requerimientos especiales y con tiempos de preparación muy cortos. Se ha aplicado con éxito en

Figura 5.

Equipo EMG wireless. Ubicación de sensores EMG. Integración con $\mathrm{MH}-$ Sensors.
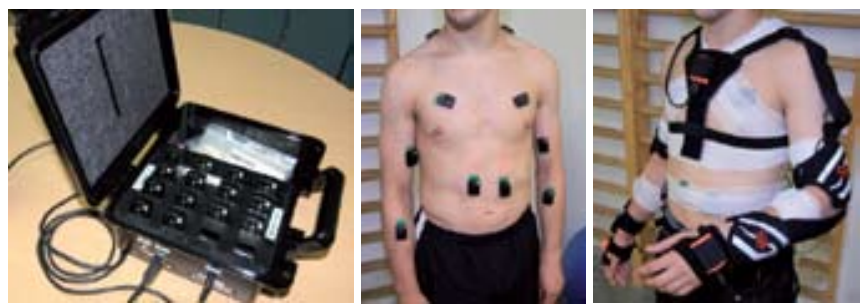
la evaluación de puestos de trabajo en entornos industriales, permitiendo aplicar distintos métodos de evaluación ergonómica.

\section{PROCEDIMIENTO DE TRABAJO}

El procedimiento a seguir previsto para llevar a cabo el análisis de riesgos de tareas repetitivas se podría sintetizar en 3 fases:

En la $1^{\text {a }}$ fase se realiza la captura del movimiento del puesto de trabajo a evaluar. Es recomendable iniciar la captura un poco antes del inicio del ciclo de fabricación y extenderla a lo largo de dos ciclos completos de fabricación.

En la $2^{\text {a }}$ fase se hace uso del software de simulación 3D donde, ejecutando ciertas funciones implementadas en dicho software, se procesarán los datos recogidos de los sensores con el fin de recrear el movimiento sobre un modelo biomecánico de hombre o mujer que se moverá como el sujeto observado (Fig. 6). A la vez, se realizaran los cálculos necesarios de ángulos y velocidades de los movimientos articulares capturados y se exportarán a ciertos ficheros para su tratamiento posterior en la siguiente fase. En la $3^{\text {a }}$ fase podremos analizar los datos resultantes en términos de riesgos en cada una de las articulaciones, junto con las valoraciones obtenidas en determinadas posturas. Finalmente podremos generar un informe detallado de los resultados del análisis.

Figura 6.

Recreación 3D del movimiento y del puesto de trabajo.
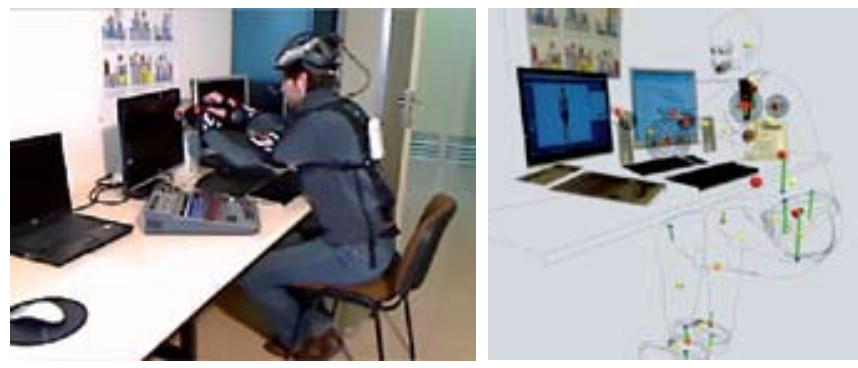

\section{DETERMINACIÓN DE LOS "INSTANTES DE RIESGO".}

Uno de aspectos que constituyen la base de la evaluación del riesgo es la determinación de los instantes o "frames" donde se produce un cambio de sentido de giro en una articulación, denominados instantes de riesgo.

Para ello, es necesario conocer la variación a lo largo del tiempo de los distintos ángulos implicados en el movimiento de una articulación. Si conocemos dicha variación en un ángulo particular (por ejemplo, la flexión del hombro), será necesario determinar los máximos y mínimos que se alcanzan a lo largo del movimiento y, precisamente, esos máximos y mínimos (que llamaremos locales) corresponderán con los cambios de sentido de giro que buscamos, en este caso, de la flexión del hombro.

En la Fig. 7 se recoge la representación gráfica de un posible ángulo de una articulación, su magnitud en función del tiempo. Los referidos cambios de giro corresponderán a un máximo o mínimo local. En el caso de la figura podemos observar dos cambios. El punto "1" se considera un máximo local del ángulo porque el valor del ángulo es superior a todos los puntos a la
Figura 7.

Determinación de máximos y mínimos locales.

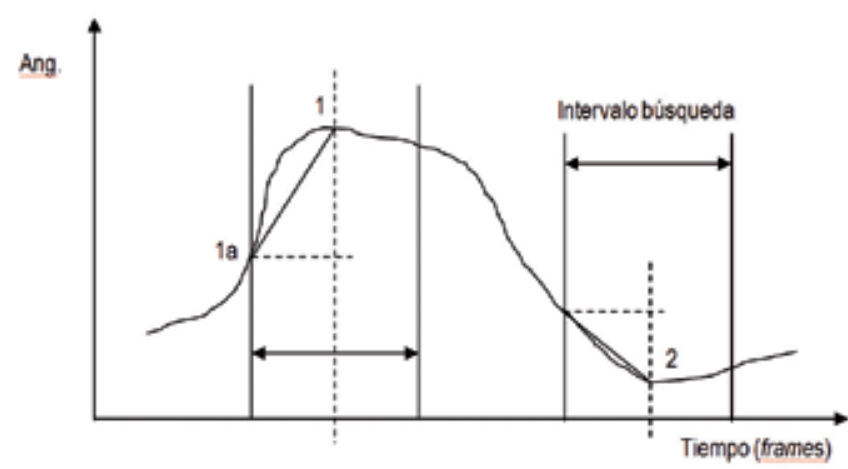

derecha e izquierda del intervalo, pero también porque la pendiente entre el punto de inicio del intervalo, punto "1a" y dicho punto "1" (velocidad angular media) es superior a un parámetro predefinido de Velocidad Angular Mínima, medido en [grados/seg]. Basta que sea suficientemente significativa en uno de los lados (aceleración o desaceleración, según el caso) para seleccionarlo como máx/mín local y, en consecuencia, como instante de riesgo. Idéntico razonamiento podemos aplicar al punto " 2 " para considerarlo mínimo local.

\section{REPRESENTACIÓN GRÁFICA DE FUERZAS Y RIESGOS}

A continuación se incluyen los distintos modos de visualización que podemos seleccionar a la hora de observar, sobre el modelo biomecánico del trabajador, el impacto de la aplicación del método de evaluación de riesgos de tareas repetitivas. La información gráfica que se muestra corresponde a los datos numéricos recogidos en los informes de salida, traducidos en vectores de fuerza y momentos aplicados, tanto en las articulaciones del modelo como en los centros de gravedad de los segmentos corporales; esto último para el caso de las fuerzas y momentos de inercia (Fig. 8). Asimismo, el valor número del riesgo resultante obtenido en cada articulación se representa con una esfera cuyo diámetro es propor-

\section{Figura 8.}

Visualización sólo de los vectores de fuerza y momentos (a). Visualización de todos los elementos, C.G. Esferas Riesgos y Vectores Fuerza (b).
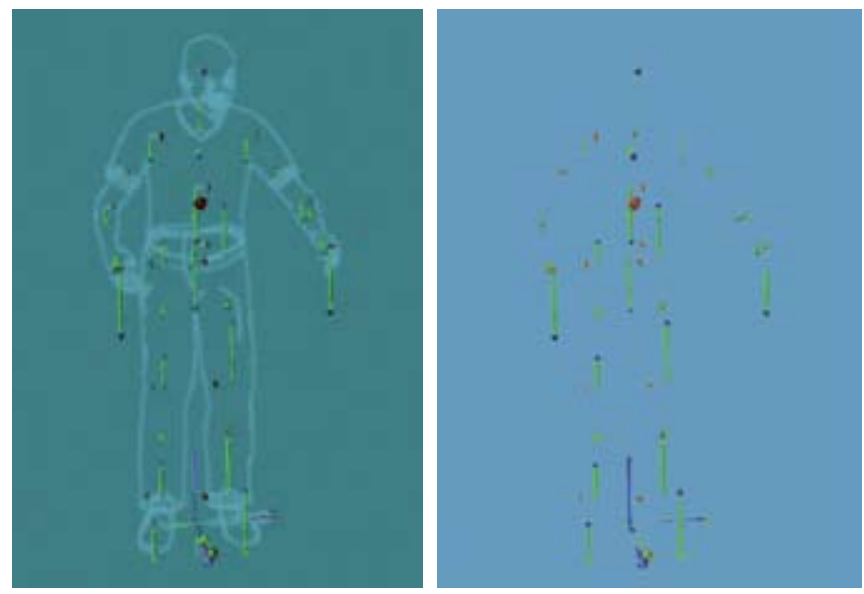
cional a dicha magnitud numérica, denominada esferas de riesgo. Permite observar, gráficamente, la variación del riesgo en cada articulación (que puede aumentar o disminuir a lo largo de la simulación), ya que corresponde al riesgo en cada instante, pero valorado en unidades de riesgo/min.

Anotar que el riesgo ponderado de todas las articulaciones de la EESS medido en [ValorRiesgo/min], se representa también con una esfera de diámetro proporcional al valor de riesgo en cada instante y su centro se ubica en la proyección del C.G. del cuerpo sobre el suelo. De esa forma se moverá con el trabajador y normalmente se situará entre los pies del modelo (Fig. 8b).

Respecto a la visualización incluida en la Fig. 8, indicar que tendremos vectores de distintos tipos:

- Fuerzas y momentos externos aplicados sobre las manos.

- De reacción provocadas sobre el modelo en los puntos de apoyo. En general son fuerzas de reacción en los pies, o bien sobre la pelvis (si está sentado) o sobre las manos si alguna de ellas está apoyada.

- Fuerzas y momentos de inercia en los C.G. de cada uno de los segmentos corporales provocados por las aceleraciones lineales y angulares en los mismos.

- Fuerzas y momentos resultantes en las articulaciones derivadas del equilibrio de fuerzas en cada instante, considerando las fuerzas anteriores.

En las siguientes figuras se recogen distintas posturas, donde se pueden visualizar los diferentes elementos citados.

En la Figura 11 se incluye el resultado del cálculo en instantes concretos de un puesto de trabajo simulado de tarea repetitiva.

\section{Figura 9.}

Sólo el pie izq. apoyado. Apoyado en ambos pies y mano izq., y mano dcha. con fuerzas externas; se puede apreciar la fuerza de reacción sobre mano izq. en dirección del antebrazo.
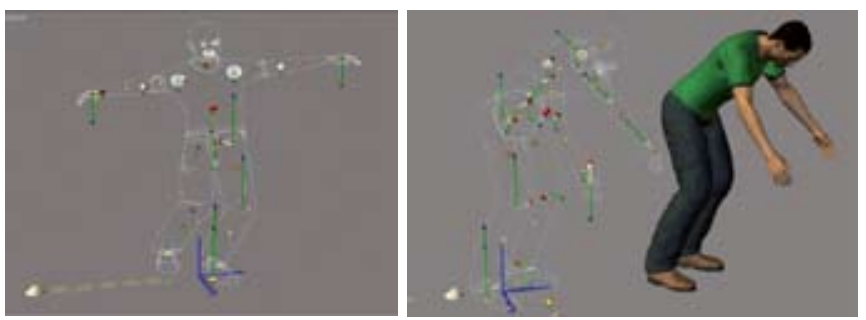

Figura 10.

Postura de sentado. Postura de silla semisentado.
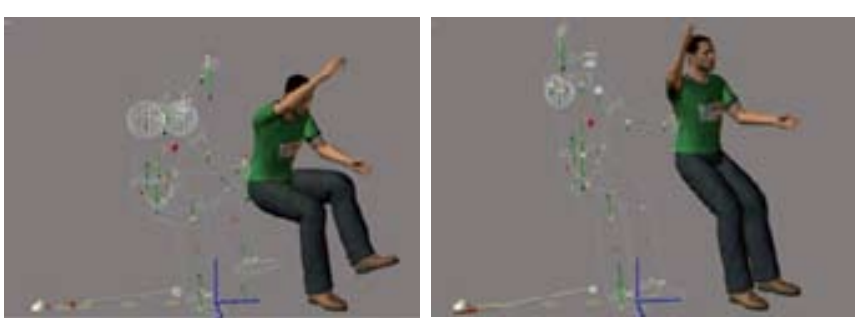

Figura 11.

Puesto simulado. Vectores de fuerza y esferas de riesgos en las articulaciones.

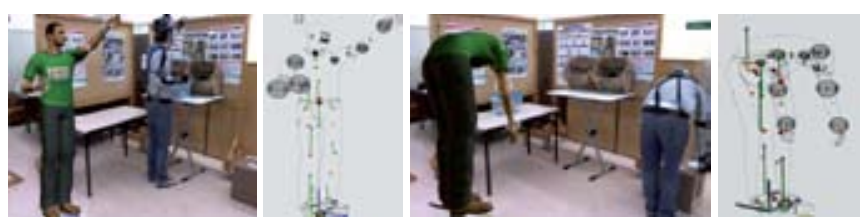

METODOLOGIA DE VALIDACION

\section{DEL MÉTODO}

Para la validación del método se ha optado por la siguiente metodología:

Se ha seleccionado una muestra representativa de puestos de trabajo donde se realicen tareas repetitivas. En concreto, se han elegido puestos de cadenas de montaje del sector de automoción y del sector de línea blanca de electrodomésticos. En dichos puestos se están llevando a cabo las siguientes tareas:

- Evaluar el riesgo con el sistema MH-Forces y con el Método OCRA (UNE-EN 1005-5), que consideramos como método "Gold Standard" a efectos de esta validación.

- Realizar un análisis estadístico de los resultados obtenidos con uno y otro método, y a diferentes niveles de detalle.

- Ajustar la parametrización del método MH-Forces acorde a los resultados obtenidos en el punto anterior, con el fin de mejorar su estimación del riesgo.

- En aquellos puestos en los que se realice manipulación manual de cargas se aplica también el método NIOSH y, en los que presenten riesgo por carga postural, el método REBA y se comparan los resultados con MH-Forces.

Para la aplicación del Sistema $\mathrm{MH}$-Forces se requiere tomar ciertas medidas antropométricos del trabajador, así como datos propios del puesto de trabajo: tiempos de ciclo de producción o turnos de trabajo, entre otros. Posteriormente, se procede a capturar el movimiento del trabajador durante la realización de la tarea haciendo uso del sistema de captura MH-Sensors, ya citado.

Para medir las fuerzas o pares externos que realiza el trabajador durante su actividad, se utiliza un equipo de Dinamometría con distintos accesorios (Fig. 12a), que nos permite medir las fuerzas de tracción, compresión y rotación que pueda llegar a realizar el trabajador con una o ambas manos. En ciertos casos también se puede hacer uso de un guante instrumentalizado para medida de presiones sobre la superficie de la mano (Fig. 12b).

Asimismo, se está aplicando el método MH-Forces en puestos de trabajo donde se había diagnosticado alguna lesión musculoesquelética en los trabajadores expuestos, al objeto de comprobar si existe correlación entre el nivel de riesgo que predice el nuevo método y la realidad constatada. Los resultados están siendo satisfactorios.

Para llevar a cabo este estudio se está contando con la colaboración del Instituto de Ergonomía Mapfre (INERMAP) y empresas de nuestro entorno del sector de automoción o de electrodomésticos como BSH-Electrodomésticos.

Figura 12.

Dinamómetro con accesorio torquímetro (a). Guante para medida de presiones (b).
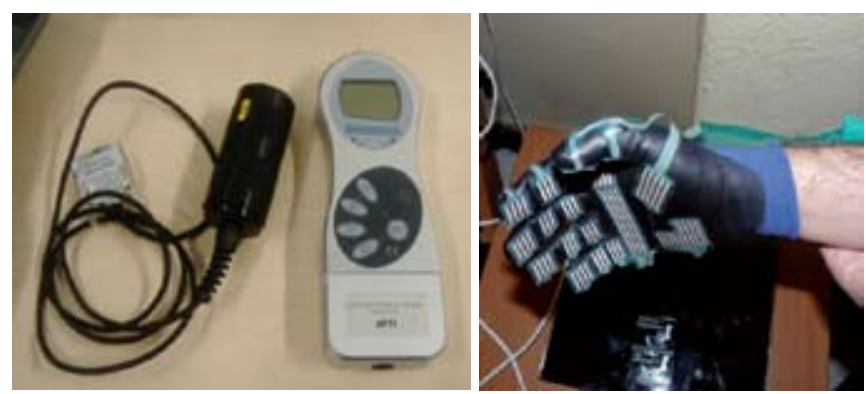
ASPECTOS DESTACADOS DEL MÉTODO

El método MH-Forces se fundamenta en contabilizar los denominados "instantes de riesgo", es decir, el número de cambios de rotación que sufren las articulaciones en cada uno de sus ejes de rotación a lo largo de la ejecución de las tareas durante el tiempo de ciclo del trabajo repetitivo. También considera como instante de riesgo una posición estática mantenida en exceso de una articulación (mantenerla inmóvil más allá de un tiempo definido, normalmente unos $6 \mathrm{seg}$ ). Detectado un cambio o postura estática en un instante concreto, calcula ciertos factores de riesgo en ese instante que serán la base de la evaluación de riesgos. Esos factores son relativos a los siguientes aspectos:

- Magnitud del ángulo de rotación donde se ha detectado el cambio de giro, así como el valor de los otros ángulos implicados en el movimiento.

- Arco de giro recorrido desde el último cambio. Un mayor arco indicará que el movimiento ha sido de gran amplitud y generalmente con menor rapidez, lo que supone menor riesgo.

- Velocidad angular alcanza poco antes, o poco después, de que la articulación se detenga. El valor de dicha velocidad indicará el grado de aceleración o desaceleración que ha sufrido la articulación, debido al cambio de sentido de rotación hasta llegar a pararse.

- Magnitudes de las fuerzas axiales y de corte, así como los momentos flectores y torsión a los que está sometida la articulación en ese instante. Mayores valores implicarán mayor riesgo para la articulación.

El valor de dichos factores se determina en función de las magnitudes medidas (ángulos, velocidades, esfuerzos) y de ciertas curvas paramétricas definidas como secuencia de puntos (valor magnitud medida y valor factor de riesgo correspondiente). Puntos intermedios se calculan por interpolación. Dichos factores de riesgo pueden variar del valor 1 hasta del orden de 1,5. La multiplicación de esos factores en un determinado cambio de rotación será el riesgo correspondiente a ese cambio (valor del riesgo).

El sistema puede detectar un riesgo por carga postural, similar a la aplicación de un método como REBA5, pero considerando también las fuerzas de inercia provocadas por el propio movimiento, y realizando un cálculo del orden de 25 posturas/seg.

En efecto, al objeto de detectar posibles posturas nocivas de una articulación en determinados instantes, en cada cambio de rotación, el informe de salida recoge el valor de riesgo del cambio, pero también su porcentaje respecto a su valor máximo, lo que nos dará un nivel de riesgo que podrá tomar los valores $(0,1,2$, 3 ó 4), que le corresponderán distintos niveles ("Sin riesgo", "Riesgo Bajo", "Riesgo Medio", "Riesgo Alto" o "Riesgo muy Alto").

Como resultado del número de cambios de giro (o posturas estáticas) detectadas en cada articulación a lo largo del ciclo de trabajo, y de las puntuaciones obtenidas de riesgo en cada caso ( 0 a 4), se puede obtener el porcentaje de veces que una articulación ha estado con riesgo 0 "sin riesgo", o con riesgo máximo 4, o bien con valores de riesgo intermedios. Esta información será relevante a efectos de valorar las articulaciones sometidas a mayor riesgo. Esto último nos da un valor añadido en relación a los sistemas de valoración tradicional de la carga postura, los cuales se limitan a determinar instantes donde el riesgo es mayor.

En relación al riesgo por manipulación de cargas, también el sistema puede dar una información interesante en este punto. La manipulación de objetos con pesos significativos pueden provocar riesgo en la zona lumbar, los cuales pueden ser también detectados con este nuevo método.

En efecto, los momentos de coger y dejar la carga coinciden normalmente con cambios en la rotación de la columna lumbar, que pueden ser detectados por el software y, en esos instantes, el sistema calcula las fuerzas axiales, tangenciales, y momentos de flexión y torsión actuando sobre dicha zona lumbar. Asimismo, en esos casos, también se tiene en cuenta las inercias provocadas por el movimiento del propio cuerpo, brazos y tórax, así como las inercias derivadas de la masa del objeto que se manipula y de las aceleraciones o deceleraciones que el trabajador realiza durante esa manipulación.

En consecuencia, aporta un valor añadido a la aplicación de métodos como $\mathrm{NIOSH}^{4}$, sin contar que también calcula momentos intermedios entre coger y dejar la carga que también pueden ser nocivos para el trabajador. Adicionalmente, tiene en cuenta con precisión la composición de los tres ángulos implicados en la columna lumbar (flexión, lateralización y rotación) que, sin duda, es importante considerar a la hora de calibrar el riesgo por manipulación de cargas.

Permite considerar esfuerzos lineales en cualquier dirección del espacio, así como momentos o pares ejercidos con las manos y en cualquier eje de rotación. Ello le confiere posibilidades de análisis más allá de las habituales de esfuerzos verticales realizados por manipulación de cargas. Adicionalmente, es posible variar esos esfuerzos o pares a lo largo del tiempo, tanto en magnitud como dirección y sentido, $\mathrm{y}$, de esa forma, permite considerar una amplia gama de situaciones que se pueden dar en la práctica. Incluso se pueden variar esas fuerzas externas para observar el efecto en la valoración del riesgo resultante y, en general, realizar un análisis de simulación de diferentes alternativas o propuestas de mejora.

Es posible contemplar que el trabajador está de pie, en bipedestación o con un solo pie apoyado, o sentado. Bastará modificar la altura de la pelvis respecto al suelo para estudiar una posición de sentado con una silla normal o bien la utilización de una silla de semisentado, donde las piernas están más estiradas, soportando parte del peso del cuerpo, y la espalda generalmente más erguida para mantener el equilibrio. También es posible considerar que tiene una o ambas manos apoyadas. Lógicamente el tipo de apoyo podrá variarse a lo largo del ciclo de fabricación.

En relación al punto anterior, sobre distintos tipos de apoyo del cuerpo, el motor de cálculo desarrollado incluye los algoritmos necesarios para estimar las reacciones en los puntos de apoyo en las distintas situaciones posibles, función de la postura en cada instante durante el movimiento, características antropométricas del trabajador y de las posibles fuerzas que ejerce con las manos; para ello, debe procurar lograr el necesario equilibro del cuerpo en cada instante. Esta estimación realizada es clave, y es la etapa previa al cálculo de esfuerzos en cada una de las articulaciones.

Como aspectos adicionales que el sistema puede contemplar, se pueden citar los siguientes:

- El tipo de agarre que se realiza con las manos al manipular objetos (sin agarre, pinza, gancho, de fuerza o palmar). La forma del agarre influirá en los riesgos resultantes sobre las muñecas. El sistema también ajustará la posición de los dedos, según el tipo de agarre seleccionado en distintos momentos de la tarea realizada por el trabajador. Ello nos ofrecerá una visión 
realista de sus movimientos, incluyendo también las manos.

- El nivel de actividad que normalmente realiza el trabajador de forma regular, que puede variar desde una situación de inactividad (valor 0) a muy activa (valor 3). Este nivel afectará a los valores máximos de esfuerzos de referencia. Para una misma tarea el riesgo resultará más elevado en trabajadores con escasa actividad física regular que en aquellos con un mayor nivel de actividad física.

- En caso de una trabajadora, las fuerzas máximas se reducen de acuerdo a un coeficiente definido por un parámetro configurable.

- El tiempo de ciclo. Este es un aspecto relevante a efectos del cálculo de riesgo por minuto, ya que los riesgos resultantes durante el tiempo entre los instantes de inicio y fin seleccionados para procesar dependen de ese tiempo. Pero con frecuencia ese tiempo capturado no coincide con el tiempo de ciclo concedido donde se consideran ciertos coeficientes de descanso. En consecuencia, el sistema permite seleccionar el tiempo a considerar en los cálculos, bien el tiempo concedido, o bien el tiempo de inicio-fin de la captura. Es recomendable el primero.

Como es obvio, para abordar el conjunto de funcionalidades y acciones expuestas, se requiere utilizar un sistema de captura de movimiento que nos permita capturar el movimiento del trabajador y trasladarlo al modelo humano de hombre y mujer, de una antropometría similar al sujeto, sobre el cual poder realizar los cálculos cinemáticos y dinámicos precisos en las articulaciones a efectos de posibilitar la estimación de riesgos en las mismos. Para tal propósito, se cuenta con el sistema Move-Human Sensor ya descrito anteriormente.

Un aspecto interesante a considerar es lo relativo a la aplicación del método en el diseño de nuevos puestos de trabajo. Haciendo uso del sistema de animación 3D de propósito general utilizado ${ }^{9}$, es razonablemente fácil reconstruir, con los personajes humanos diseñados, las posturas claves que el trabajador debe adoptar en el nuevo puesto de trabajo, incluso considerando distintos percentiles antropométricos. Las posturas intermedias son calculadas por interpolación obteniendo un movimiento similar al generado desde un sistema de captura de movimiento.

Consecuentemente, aunque el puesto de trabajo aún no exista, se puede aplicar el mismo método de cálculo para evaluar los potenciales riesgos ergonómicos y, en caso de un resultado no satisfactorio, realizar los cambios oportunos en el puesto o en la operativa de trabajo hasta lograr que los riesgos previstos se sitúen en límites admisibles. Acciones tomadas en una etapa de diseño pueden suponer sin duda un ahorro de costes muy significativo. La colaboración entre los ingenieros de diseño y los técnicos de prevención haciendo uso de este sistema sería muy positiva.

\section{CONCLUSIONES}

El método MH-Forces expuesto ha tratado de superar las limitaciones de los métodos actuales dirigidos a tal propósito. Por una parte, en lo relativo a proporcionar un sistema de fácil aplicación liberando al técnico de prevención de tareas tediosas y, por otro lado, al tratarse de un sistema automatizado de medida del riesgo en las articulaciones, no está influenciado por la subjetividad del evaluador.

Se considera que disponer de este nuevo método de valoración de riesgos supone un avance significativo respecto a los métodos de evaluación ergonómico actuales y puede constituirse en una herramienta avanzada de apoyo en el ámbito de la evaluación de riesgos musculoesqueléticos, y de interés para técnicos en prevención de riesgos laborales, médicos del trabajo, fisioterapeutas y terapeutas en salud ocupacional y, en general, para técnicos involucrados en disciplinas afines con la ergonomía o la biomecánica.

En el ámbito de la prevención de riesgos laborales se considera que este método podrá contribuir a facilitar las condiciones de trabajo y lograr una mejora de la calidad de la vida y salud laboral de los trabajadores implicados, así como efectos positivos $\mathrm{y}$ tangibles en términos de productividad para las empresas.

\section{AGRADECIMIENTOS}

Sincero agradecimiento al Instituto de Investigación en Ingeniería de Aragón (I3A) de la Universidad de Zaragoza, por los medios aportados, tanto materiales como humanos, y con especial mención a Dña. Isabel García, técnico del Laboratorio de Biomecánica del citado Instituto. A las empresas colaboradoras como BSH-Electrodomésticos España, Plantas de la Cartuja y Montañana de Zaragoza. Asimismo, al Instituto de Ergonomía Mapfre (INERMAP) y a su Dtor. D. José Manuel Álvarez por los consejos aportados durante la realización de este proyecto de investigación. 
1. España. Asociación Española de Normalización y Certificación. Norma UNE-EN 1005-3. Seguridad de las máquinas; Comportamiento fisico del ser humano. Parte 3: Limites de fuerza recomendados para la utilización de máquinas [online]. Madrid: AENOR; 2002. [citado 2 Jul 2009]. Disponible en: http://www.upf.edu/materials/bib/docs/iula/UNE1005.pdf

2. Chafin B, Anderson GB, Martin BJ. Occupational Biomechanics 3rd ed. New York: Wiley Interscience; 1999.

3. Álvarez $E$, Hernández $A$, Tello $S$. Manual de evaluación de riesgos para la prevención de trastornos musculoesqueléticos. Madrid: Factors Humans; 2009.

4. Waters Putz-Anderson G. Aplicación Manual for the Revised NIOSH Lifting Equation. Atlanta: Centers for Disease Control and Prevention; 1994.

5. Nogareda S. NTP 601; Evaluación de las condiciones de trabajo: carga postural. Método REBA (Rapid Entire Body Assessment)[en línea]. Madrid: Instituto Nacional de Seguridad e Higiene en el Trabajo; 2003[consultado may 2013]. Disponible en: http://www.insht.es/InshtWeb/Contenidos/ Documentacion/FichasTecnicas/NTP/Ficheros/601a700/ntp_601.pdf

6. Hignett S, McATAMNEY L. Rapid Entire Body Assessment: REBA. Appl Ergon. 2000;31: 201-205.

7. España. Asociación Española de Normalización y Certificación. UNE-EN 1005-5:2007[en línea]. Seguridad de las máquinas. Comportamiento físico del ser humano. Parte 5: Evaluación del riesgo por manipulación repetitiva de alta frecuencia. Madrid: AENOR; 2007. [citado jul 2013]. Disponible en: http://www.aenor.es/aenor/normas/normas/fichanorma.asp?codigo= N0040129\&tipo=N\#.Uf6P7dLZaEw

8. Colombini D, Occhipinti E, Griego A. Evaluación y Gestión del Riesgo por Movimientos Repetitivos de las Extremidades Superior. Santiago de Chile: ACHS; 2004. Colección Cátedra Mutual CYCLOPS UPC.

9. Xsens Motion Technologies [on line]. Enschede ( Netherlands): XSENS; 2013 [citado mar 2013]. Disponible en: www.xsens.com

10. Software animación 3D Poser 8 [on line]. Aliso Viejo: Smith Micro Software Inc; 2013[citado mar 2013]. Disponible en: http://poser.smithmicro.com/

11. Houston Ronald L. Principles of Biomechanics. London: CRC Press-Taylor \& Francis Group; 2009.
12. Nordin M, Frankel V. Biomecánica básica del sistema musculoesquelético. Madrid: McGraw-Hill Interamericana; 2004.

13. González C. Mecánica del Sólido Rígido. Barcelona: Ariel; 2003.

14. Marín JJ, Boné MJ, Ros R, Martínez M. Move-Human Sensors: Sistema portátil de captura de movimiento humano basado en sensores inerciales, para el análisis de lesiones musculoesqueléticas y utilizable en entornos reales. En: $6^{\circ}$ Congreso Internacional de Prevención de Riesgos Laborales; La Coruña, mayo 2008. Madrid: ORP; 2008.

\section{Referencias complementarias:}

Barrientos A, Felipe L, Balaguer C, Aracil R. Fundamentos de Robótica. Madrid: McGraw Hill Interamericana; 2007.

Burbano S, Burbano E, Gracia C. Física General. 32a ed. Madrid: Tébar; 2007.

España. Ministerio de trabajo y asuntos sociales. Real Decreto 1971/1999, de 23 de diciembre, de procedimiento para el reconocimiento, declaración y calificación del grado de minusvalía. BOE. (22) 26 ene 2000.

España. Jefatura del Estado. Ley 34/2003 de 4 de noviembre de modificación y adaptación a la normativa comunitaria de la legislación de seguros privados. BOE. (265) 5 nov 2003.

España. Ministerio de la Presidencia. Real Decreto Legislativo 8/2004, de 29 de octubre, por el que se aprueba el texto refundido de la Ley sobre responsabilidad civil y seguro en la circulación de vehículos a motor. BOE. (267) de 05 nov 2004.

Marín JJ, García I, Gil C, Boné MJ. Validación de sistema de medición de capacidad funcional basado en sensores inerciales. En: XXVIII Congreso de la Sociedad Española de Ingeniería Biomédica; Madrid 24-26 nov. 2010. Madrid: Universidad Carlos III, 2011.

NIOSH. Work practices guide for manual handling. Cincinnati: US Department of Health and Human Services. National Institute for Occupational Health; 1981. Technical Report, 81122.

Ollero A. Robótica Manipuladores y Robots Móviles. Barcelona: Marcombo; 2001.

U.S.A. American Medical Association (AMA) Guias para la evaluación de las deficiencias permanentes. Madrid: Ministerio de Asuntos Sociales; 1994. 